\section{Rapid Quantification of Cell Numbers Using Computer Images}

\section{BioTechniques 28:624-628 (April 2000)}

The quantification of cell numbers is a standard measure for proliferation and is often used to interpret tissue culture experiments. However, counting manually using a microscope or microscopic images is time consuming. In this paper, we present a novel method for rapid quantification of nuclei numbers using fluorescent immunohistochemical staining and conventional imaging software. This technique is easily applicable, reliable and a desirable alternative to manual cell counting. In addition, we show that the technique can be used to analyze the differentiation rate of skeletal muscle cells.

To determine the feasibility of using pixel quantification as an alternative to manual nuclei counting, we used proliferating $\mathrm{BC} 3 \mathrm{H} 1$ skeletal muscle cells, which proliferate in high serum medium (7). By assaying cell numbers in proliferating cultures, we show that manual counting of nuclei can be replaced by simple pixel quantification of nuclei staining. At various time points after plating, cultures of $\mathrm{BC} 3 \mathrm{H} 1$ cells were fixed and stained using Hoechst 33342 dye (Sigma, St. Louis, MO, USA).

Images of the cultures were captured and post-processed in Adobe ${ }^{\circledR}$ Photoshop ${ }^{\circledR}$. The images were adjusted with the Level function to remove background and create a uniform staining. Using the Threshold function, we converted an image to a two-bit image. Figure 1A displays an image captured by the charge-coupled device (CCD) camera (Sony, New York, NY, USA). Figure 1B displays the same image after level adjustment and conversion to a two-bit format. Using the Select Color function, the nuclei staining was selected and the number of pixels was determined by the Histogram function.

By comparing the number of nuclei to the number of pixels in five images, an average number of pixels per nuclei was determined. This conversion factor was used to determine the average number of nuclei at five different time points of the $\mathrm{BC} 3 \mathrm{H} 1$ cultures, using five images for each time point. Figure $1 \mathrm{C}$ demonstrates that manual counting and pixel numbers show a similar increase in cell numbers as the cultures proliferate from very low density to confluency. Figure 1D establishes a linear correlation between manually counted nuclei numbers and numbers based on converted pixel values. It should be noted that the cells decrease in size as they reach confluency, which introduces a small variation between the two methods of cell counting. Linear regression between the two meth- ods is significant and proves the validity of our method (Figure 1D).

Having shown that pixel quantification can be used to determine the number of nuclei in cultures, we investigated whether the technique could be used to analyze the amount of myosin staining and differentiation in muscle cultures. Normally with images of differentiated cell cultures, differentiation is measured by determining the number of myosin-positive cells or nuclei. Here, we show that quantifying the number of myosin-positive pixels is comparable to these techniques. To evaluate this, we

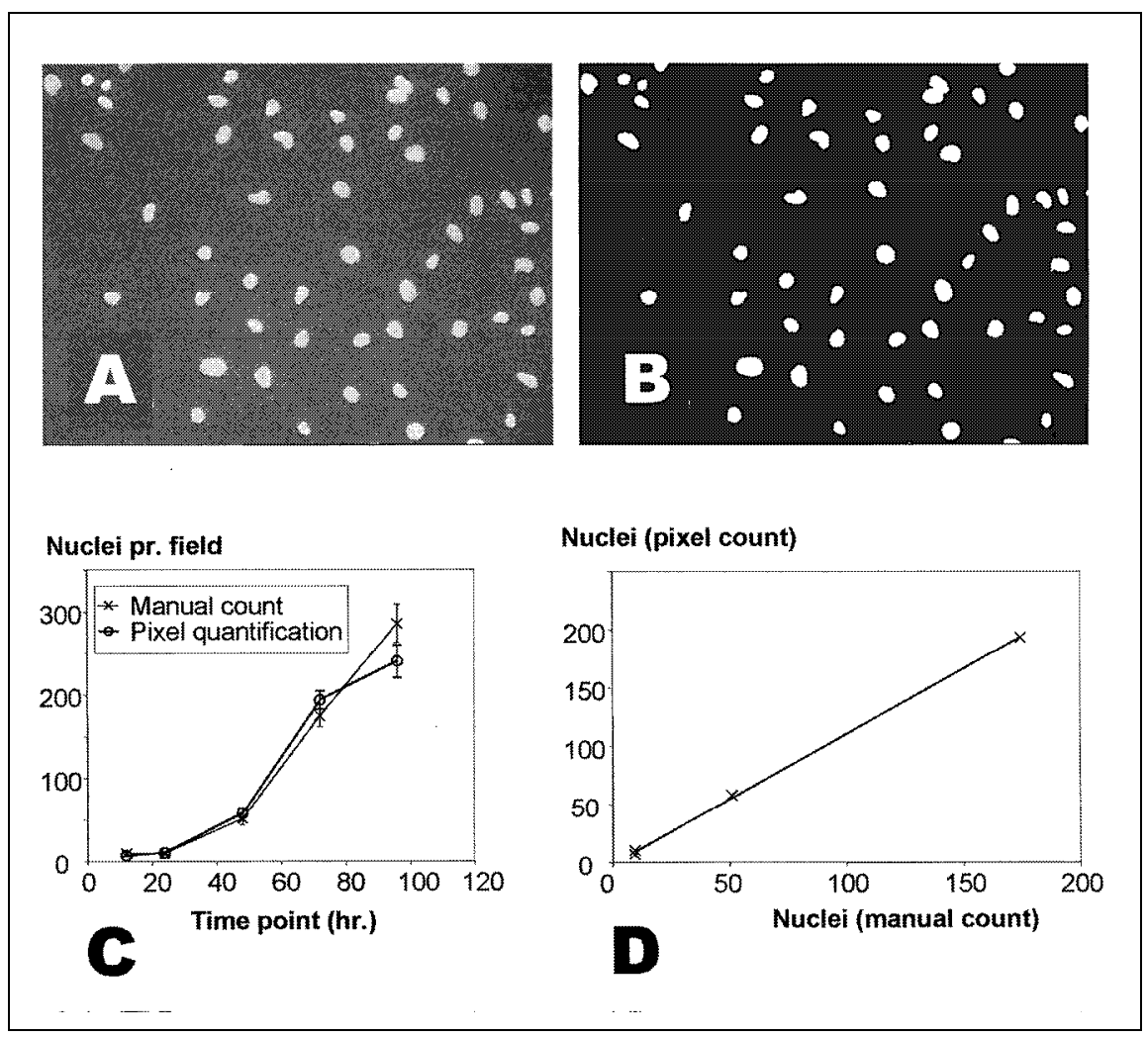

Figure 1. Comparison of manual counting and pixel quantification of nuclei in proliferating BC3H1 cultures. Skeletal muscle $\mathrm{BC} 3 \mathrm{H} 1$ cells were grown at $37^{\circ} \mathrm{C}, 5 \% \mathrm{CO}_{2}$ in $30 \mathrm{~mm}$ plates in a proliferation medium consisting of Basal Medium Eagle (BME), penicillin and streptomycin (all from Life Technologies, Gaithersburg, MD, USA) and 15\% fetal bovine serum (HyClone Laboratories, Logan, UT, USA). Cells were fixed with methanol at various time points to assay proliferation of the cultures. Nuclei were stained using $1 \mathrm{~mL}$ Hoechst 33342 dye per plate $(1: 1000$ dilution of a $10 \mathrm{mg} / \mathrm{mL}$ stock solution in PBS, $\left.138 \mathrm{mM} \mathrm{NaCl}, 2.7 \mathrm{mM} \mathrm{KCl}, 8.1 \mathrm{mM} \mathrm{Na}_{2} \mathrm{HPO}_{4}, 1.2 \mathrm{mM} \mathrm{KH}_{2} \mathrm{PO}_{4}\right)$ and washed twice in PBS and once in double-distilled water before the culture plates were mounted with coverslips using FluorSave (Calbiochem-Novabiochem, San Diego, CA, USA). Staining was visualized with UV light under a fluorescent microscope and digitally captured using a Sony CCD camera and image-capturing software (Northern Eclipse, Empix Imaging, Mississauga, ON, Canada). The original picture (A) was adjusted in Adobe Photoshop using the Levels function to sharpen Hoechst-positive pixels and converted to a twocolor image (B). All positive pixels were selected using Select Color and the number of pixels was recorded using Histogram. A pixel-to-nuclei number conversion factor was determined for the pixel counts by comparing pre-counted cultures with pixel numbers. (C) Both methods of nuclei counting show a similar increase in cell numbers. (D) Manual counts of nuclei and nuclei numbers determined by pixel quantification for 12-72 h time points display a linear correlation between the two methods $\left(\mathrm{R}^{2}=0.9995, P<0.0002\right)$. Data points represent averages of 5 images. Error bars indicate SEM. 
used a $\mathrm{BC} 3 \mathrm{H} 1$ cell de-differentiation assay. The de-differentiation ability of $\mathrm{BC} 3 \mathrm{H} 1$ cells is unique to these cells and has been described extensively $(2-6,8)$. With serum stimulation, differentiated $\mathrm{BC} 3 \mathrm{H} 1$ cells lose muscle specific protein expression and reenter the cell cycle. A quantitative decrease in myosin staining is observed. $\mathrm{BC} 3 \mathrm{H} 1$ cells were differentiated and treated with growth medium for various time periods.

The cultures were fixed at different time points and stained for myosin expression using an anti-myosin primary antibody and a TexasRed ${ }^{\mathrm{TM}}$-coupled secondary antibody. Images displaying myosin/TexasRed-positive cells were captured and post-processed in Adobe Photoshop. To determine the amount of myosin/TexasRed positive pixels, the images were adjusted using the Levels function to remove background staining. Using the Select Color function, red pixels were selected and quantified
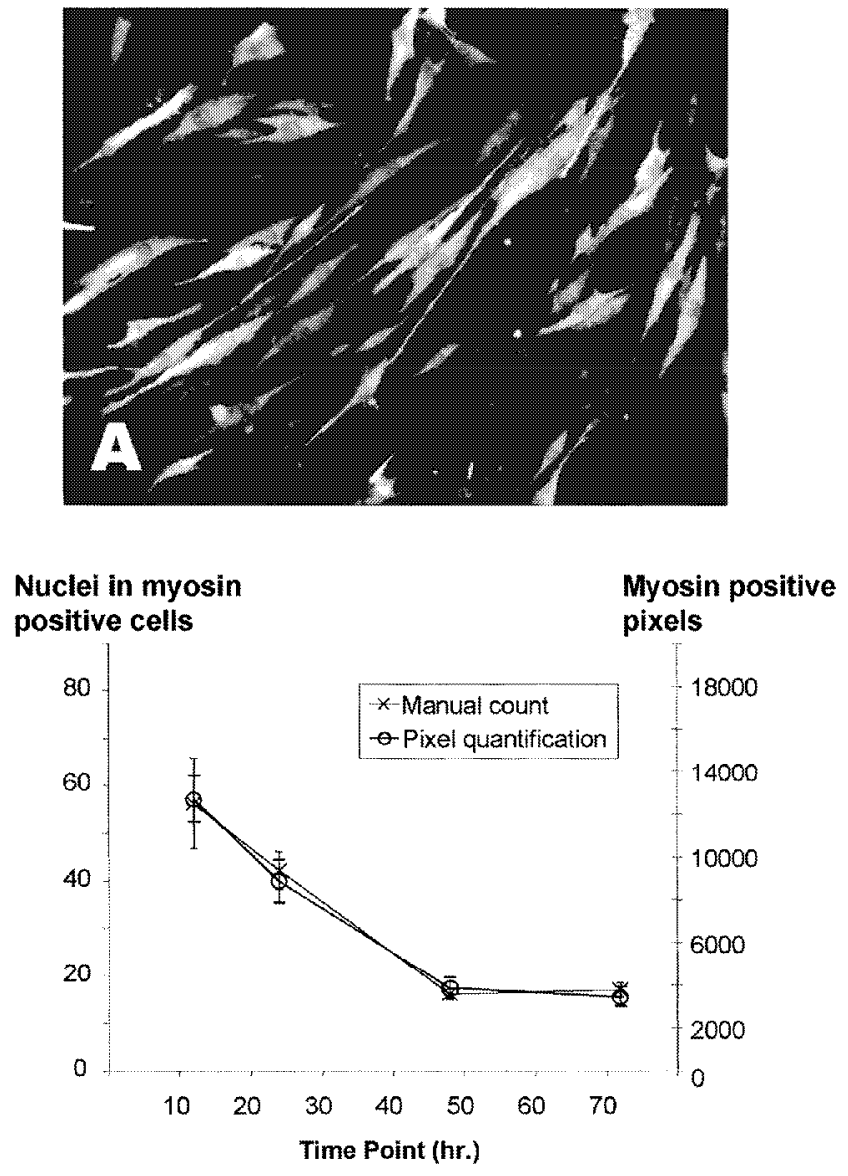

Figure 2. Myosin-positive pixels as a valid measure for differentiation. Differentiation of the $\mathrm{BC} 3 \mathrm{H} 1$ cells was accomplished by switching cells to a differentiation medium (BME, penicillin/streptomycin, $0.5 \%$ FBS). De-differentiation assays were performed following four days of differentiation by feeding cells growth medium. Visualization of differentiated $\mathrm{BC} 3 \mathrm{H} 1$ cells was performed using immunofluorescence. Following fixation in methanol, plates were rinsed twice with PBS and incubated in PBS + 5\% goat serum for $1 \mathrm{~h}$ at $22^{\circ} \mathrm{C}$. Cells were then sequentially incubated with: $(i)$ anti-myosin antibody (MF-20; Developmental Studies Hybridoma Bank (1) diluted 1:10 in PBS + 1\% goat serum; (ii) TexasRed-conjugated goat anti-mouse IgG2b (Fisher, Pittsburgh, PA, USA) diluted 1:100 in PBS + 1\% goat serum; and (iii) Hoechst 33342 dye as described in Figure 1. All incubations were for $1 \mathrm{~h}$ at $22^{\circ} \mathrm{C}$. TexasRed images were captured using a TexasRed filter $(596 \mathrm{~nm})$. All other imaging was performed as described in Figure 1. (A) Example of TexasRed/myosin-stained BC3H1 cultures. (B) At four time points, the number of nuclei in myosin-positive cells as a percentage of the total nuclei number and the number of red (myosin-positive) pixels per total number of nuclei were compared. Linear correlation between the two methods was significant $\left(\mathrm{R}^{2}=0.9924, P<0.003\right)$. Data points represent averages of five images. Error bars indicate SEM.

using the Histogram function. Figure 2A shows an example of a differentiated culture. Figure 2B shows differentiation as measured by manual counting of myosin-positive nuclei and quantification of myosin-positive pixels at four time points after de-differentiation. The results show that pixel quantification yields results that are identical to manual counting and can be used to compare differentiation levels of cell cultures.

Manual counting of cell numbers is a laborious but necessary process that is needed to interpret cell culture experiments. The technique described in this paper is a fast and reliable alternative. A comparison of the time used by the two methods shows that manual counting of the cell described in Figure 1 took $90 \mathrm{~min}$, while pixel quantification was completed in $15 \mathrm{~min}$. Clearly, this difference illustrates the advantage of our technique.

Furthermore, pixel quantification of nuclei can be performed on any nuclei staining. The example used here is with Hoechst 33342 staining but with adequate images, the same technique could be used for other fluorescent or colorimetric staining. The requirements are nuclei-specific staining, either general nuclei staining or proliferating nuclei only (i.e., BrdU staining) and a consistent imaging process. The latter aspect is important because variations in staining between samples will cause a variation in pixel numbers per nuclei and introduce an error in nuclei numbers.

In addition, we show that the number of myosin-positive pixels in images can be used as a measure of differentiation levels, which correspond to differentiation levels assayed by the traditional technique of counting myosin-positive cells. This is a novel and easily applied technique that yields quick and reproducible results.

\section{REFERENCES}

1.Bader, D., T. Masaki and D.A. Fischman. 1982. Immunochemical analysis of myosin heavy chain during avian myogenesis in vivo and in vitro. J. Cell Biol. 95:763-770.

2.Kelvin, D.J., G. Simard and J.A. Connolly. 1989. FGF and EGF act synergistically to induce proliferation in $\mathrm{BC} 3 \mathrm{H} 1$ myoblasts. J. Cell. Physiol. 138:267-272.

3.Lathrop, B., E. Olson and L. Glaser. 1985. Control by fibroblast growth factor of differ- 
entiation in the $\mathrm{BC} 3 \mathrm{H} 1$ muscle cell line. $\mathrm{J}$. Cell Biol. 100:1540-1547.

4.Lathrop, B., K. Thomas and L. Glaser. 1985. Control of myogenic differentiation by fibroblast growth factor is mediated by position in the G1 phase of the cell cycle. J. Cell Biol. 101:2194-2198.

5.Olson, E.N., K.L. Caldwell, J.I. Gordon and L. Glaser. 1983. Regulation of creatine phosphokinase expression during differentiation of BC3H1 cells. J. Biol. Chem. 258:2644-2652.

6.Olson, E.N., E. Sternberg, J.S. Hu, G. Spizz and C. Wilcox. 1986. Regulation of myogenic differentiation by type beta transforming growth factor. J. Cell Biol. 103:17992805.

7.Schubert, D., A.J. Harris, C.E. Devine and S. Heinemann. 1974. Characterization of a unique muscle cell line. J. Cell Biol. 61:398413.

8.Spizz, G., D. Roman, A. Strauss and E.N. Olson. 1986. Serum and fibroblast growth factor inhibit myogenic differentiation through a mechanism dependent on protein synthesis and independent of cell proliferation. J. Biol. Chem. 261:9483-9488.

Address correspondence to Dr. Kevin Hannon, BMS Dept., School of Veterinary Medicine, Purdue University, West Lafayette, IN 47907, USA. Internet: kmh@vet. purdue.edu

Received 13 September 1999; accepted 17 December 1999.

Thomas Steenstrup, Kari L. Clase and Kevin M. Hannon School of Veterinary Medicine Purdue University West Lafayette, IN, USA

\section{Sequencing Difficult DNA Templates Using Membrane-Mediated Loading with Hot Sample Application}

BioTechniques 28:628-630 (April 2000)

One of the most frequent problems during PAGE-based DNA sequencing is the occurrence of band compression. It manifests itself in the alteration of the regular spacing between bands in sequencing gel banding patterns and/or peak overlap on automated sequencing data (electropherograms). Two or more adjacent bands can appear at the same position, resulting in discontinuous and/or incorrect sequence calls. The reason for these compression artifacts is that DNA fragments form strong secondary structures. This intramolecular base-pairing usually forms hairpin motifs within the GC-rich regions of the DNA templates (7). These compact structures migrate faster because of their higher electrophoretic mobility (smaller hydrodynamic volume) than that of the normal chains, which causes possible co-migration with shorter fragments without loops.

In the past decade, attempts have been made to eliminate this problem by using strong denaturing conditions during gel electrophoretic separation. This can be accomplished by using higher concentrations of urea (7-9 M) or additional denaturing agents, such as formamide in the sequencing gel, in addition to running the gel at an elevated temperature, typically higher than $50^{\circ} \mathrm{C}$ (3). Replacing dGTP with nucleotide analogs, such as dITP or 7-deaza$\mathrm{dGTP}$, is another option to prevent band compression by decreasing the tendency of hydrogen bond formation $(4,6)$.

Here, we present a simple solution to keep secondary structures denatured during the sample application step. To prevent possible renaturation in our protocol, the hot reaction mixture was loaded onto a preheated membrane loader (1), immediately following the heat denaturation step after the sequencing reaction. Once the DNA fragments were on the membrane, they stayed denatured and did not migrate until the electric field was applied (1). PAGE was then started with high voltage at room temperature. Using this hot sample application method and high-voltage run, DNA sequencing fragments seem to remain in their unfolded conformation during the separation process, which consequently eliminates any band compression. During the electrophoresis separation, there was no need to heat the separation platform (2), which avoided the risk of matrix instability (5).

The benefit of this hot-loading process over regular loading is illustrat- ed in Figure 1, where M13mp18 ssDNA was used as a model sequence. In Figure $1 \mathrm{~B}$, one band in the $\mathrm{G}$ line shows the unusual mobility at the compressed site, which leads to the sequence of $5^{\prime}$ CGGGTACCG $-3^{\prime}$, where $\underline{\mathrm{CCG}}$ folds around and tends to pair with $\underline{\mathrm{CGG}}$. This GTA-loop hairpin with the $3 \mathrm{G}-\mathrm{C}$ pair stem, observed under regular loading conditions (Figure 1B), was successfully eliminated by our hot sample application technique in conjunction with membrane-mediated loading (Figure 1A). Another advantage of our method is the ease of loading the sequencing samples onto the tabs of the membrane loader.

The Thermo Sequenase ${ }^{\mathrm{TM}}$ fluorescent-labeled primer cycle sequencing kit was purchased from Amersham Pharmacia Biotech (Piscataway, NJ, USA). One nanogram of M13mp18 ssDNA (New England Biolabs, Beverly, MA, USA) and 1 pM of FluoroTide $^{\text {TM }}$ M13 pUC(-21) Bodipy ${ }^{\circledR}$-TMR labeled primer (Molecular Probes, Eugene, OR, USA) were added to $2 \mu \mathrm{L}$ of each $\mathrm{G}, \mathrm{A}, \mathrm{T}$ and $\mathrm{C}$ reagents, respectively. Cycle sequencing reactions were accomplished in an Easy Cycler ${ }^{\mathrm{TM}}$ power block system (Ericomp, San Diego, CA, USA) using 20 cycles of 30

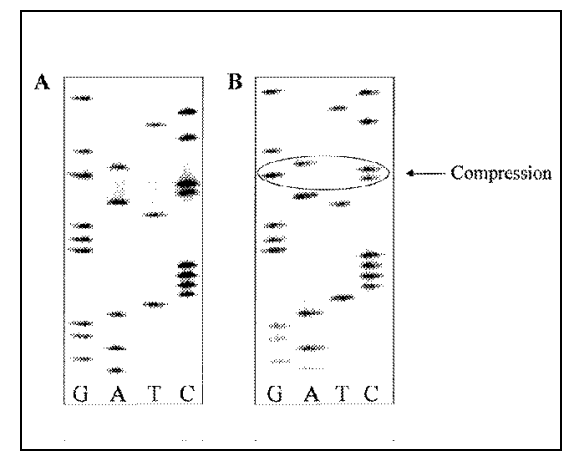

Figure 1. Hot vs. ambient temperature sample application in conjunction with membrane mediated loading of DNA sequencing gels. Sequencing with (A) or without (B) the hot sample application technique. A critical part of the sequence of M13mp18 single-stranded DNA is shown to illustrate the compression (B) and the elimination of compression by the hot sample application method (A). The samples were heated for $2.5 \mathrm{~min}$ at $95^{\circ} \mathrm{C}$, and $0.5 \mu \mathrm{L}$ aliquots of each reaction mixture were either immediately spotted onto the tabs of a preheated $\left(95^{\circ} \mathrm{C}\right)$ membrane loader (A) or cooled down in an ice-cold water bath before spotting (B). Electrophoresis separations were performed at room temperature by applying a $75 \mathrm{~V} / \mathrm{cm}$ electric field strength. 